\title{
DFT Calculations of the Neighboring Groups Effects on Cheletropic Reaction of 2,5-Dihydrothiophene Sulfone
}

\author{
Zahra Javanshir $^{\text {a*}, \text { Saeed Jameh-Bozorghi }}{ }^{\mathrm{b}}$, Parasto Peykic \\ ${ }^{a}$ Department of chemistry, faculty of science, Ahar branch, Islamic Azad University, Ahar, Iran \\ ${ }^{b}$ Department of chemistry, faculty of science, Hamedan branch, Islamic Azad university, Hamedan, Iran \\ ${ }^{c}$ Department of chemistry, faculty of science, Arak branch, Islamic Azad university, Arak, Iran \\ *Corresponding author's E-mail address: z_chem2005@yahoo.com, Tel.: +989149869713
}

Received: 21 October 2018, Revised: 5 November 2018, Accepted: 25 November 2018

\begin{abstract}
A B S T R A C T
In this research DFT calculations were used to investigate the neighboring effects on cheletropic reaction of 2,5-Dihydrothiophene sulfone (1). Structural properties and stereoelectronic behavior in the activation energy and enthalpy of cheletropic reaction were performed for 2,5-Dihydrothiophene sulfone where substitutions of $\mathrm{CF}_{3} ; \mathrm{CCl}_{3}$ and $\mathrm{CBr}_{3}$ that were placed on Sulfolen were investigated using DFT-B3LYP/6-311+ $\mathrm{G}^{* *}$ level of theory. The Results of calculations using B3LYP, HF and MP2 [basic 6-311+G**] showed that the trend of electronic effects on the cis and trans state is different. Reaction rate and activation energy in cis compounds showed that increasing electronegativity and decreasing the band length reduced the reaction speed. Trend of reaction rate and activation energy in trans compounds showed that in cheletropic reaction rate and activation energy not only electronic effects but also the Steric effects play a main role. DFT/B3LYP/6-311+G** calculation results also confirm these results. In cis compounds, 2,5- three Floro metyl Sulfolen (2) has the highest activation energy about (12.25 kcal/mol) and the lowest rate of reaction. In trans compounds, Trans-2,5- three chloro metyl Sulfolen (7) has the highest activation energy about $(12.99 \mathrm{kcal} / \mathrm{mol})$ and the lowest rate of reaction.
\end{abstract}

Keywords: DFT; cheletropy; 2,5-Dihydrothiophene sulfone; NBO; stereo-electronic effect.

\section{G R A P H I C A L A B S T RA C T}

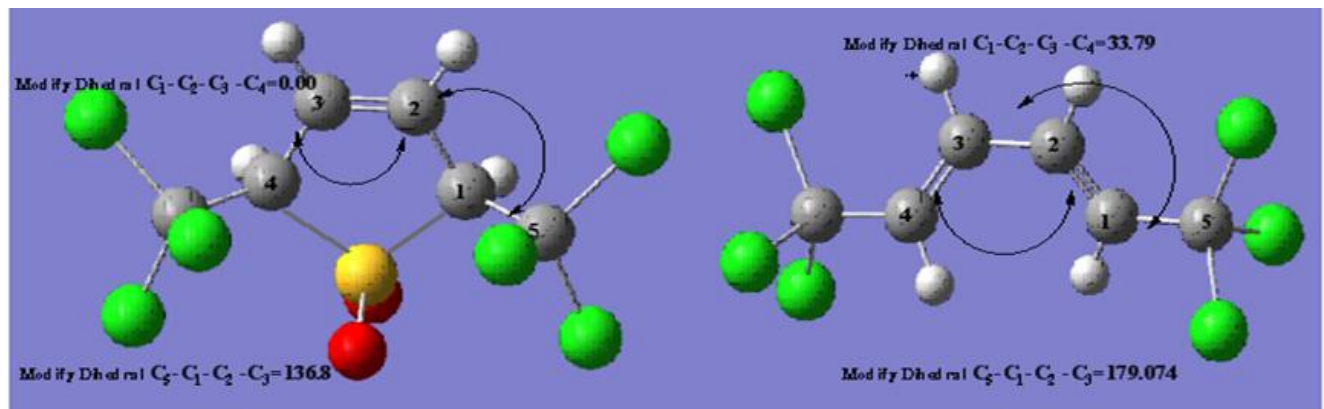




\section{Introduction}

The sulfur dioxide gas is primarily used for the production of sulfuric acid, as an oxidising agent to reduce hydrogen sulfide to elemental sulfur, and even as a fruit preservative [1]. Woodward and Hoffmann Cheletropic reactions are defined as precyclic reactions in which two or more linked $\sigma$ bond in an atom form and break [2]. Specifically, cheletropic reactions are a subclass of cycloadditions. The only difference is that on one of the reagents, both new bonds are being made to the same atom [3]. To study the molecular mechanisms of a wide range of organic reactions been investigated, such as Cope rearrangements, Diels-Alder, 1,3-dipolar and other cycloadditions, the Friedel-Crafts reaction, the Staudinger reaction [4-16].

DFT, nowadays, plays the role of a computational workhorse used by, e.g., most practicing organic chemists when investigating reaction paths and calculating reaction and activation energies [17]

In the study by Pablo Jaque at al., it was concluded that the WH rules, essentially based on orbital and wave function symmetry, can be retrieved in a "densityonly" context using conceptualDFT-based descriptors. This finding highlights the basic role of the electron density, which, despite of its much simpler mathematical form (function of three variables) as compared to the wave function (function of $4 \mathrm{~N}$ variables), is shown to carry the necessary information to regain the celebrated WH rules based on the symmetry of the wave function [18].

In this paper, original results of DFT quantum mechanical methods to study the effects of electron-electron band were used. Reactive compounds 1 to 7 are:

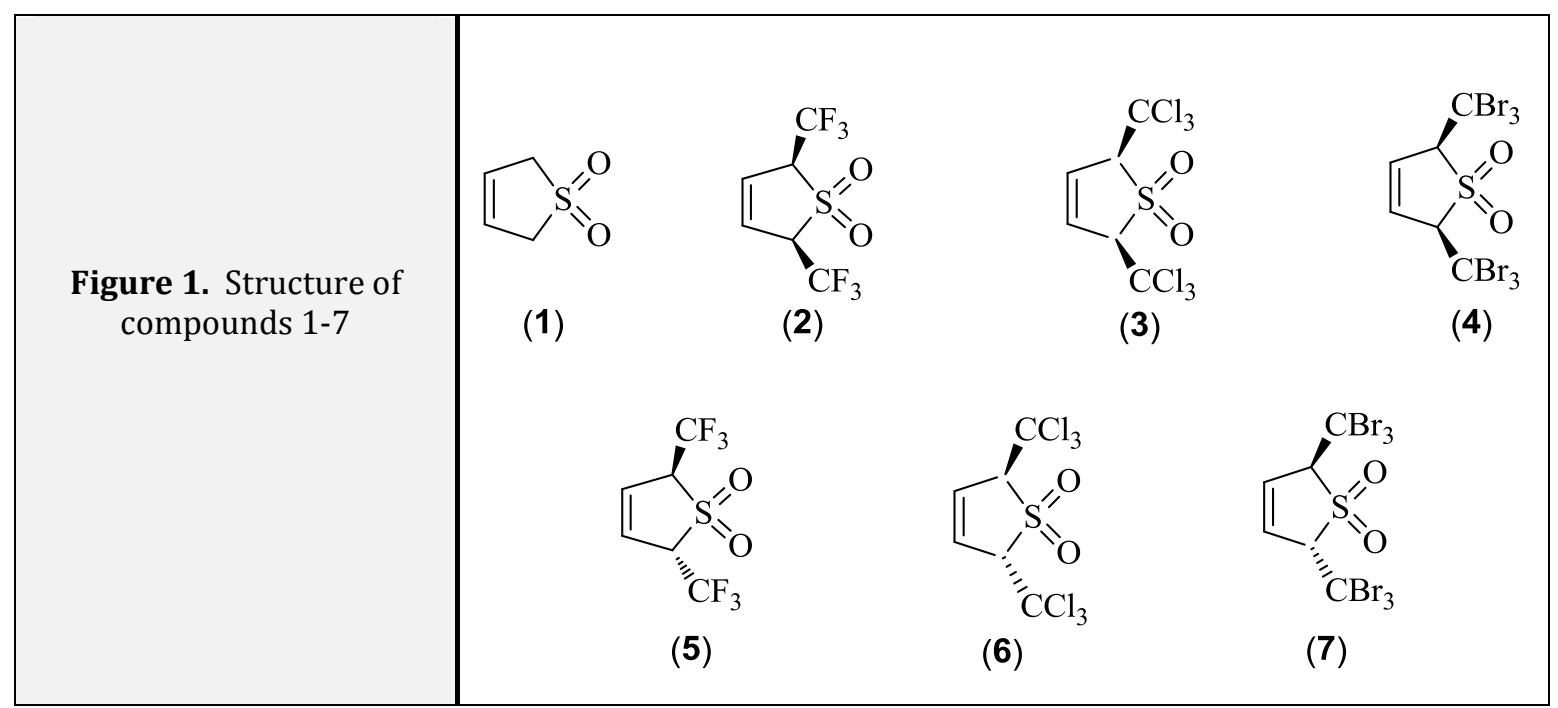




\section{Methods and Calculation details}

$\mathrm{Ab}$ initio molecular orbital (MO) calculations were carried out using B3LYP/6-311+G** level of theory with the GAUSSIAN 2003 package of programs [19] on a personal computer.

The basis set and method dependencies were probed by single point energies computed at B3LYP/6-311+G*, $\mathrm{HF} / 6$ $311+\mathrm{G}^{* *}, \quad \mathrm{MP} 2 / 6-311+\mathrm{G}^{* *} \quad$ optimized geometries.

Energy-minimum molecular geometries were located by minimizing energy with respect to all geometrical coordinates without imposing any structurally symmetrical constraints and transition state structures were obtained by QST2 subroutine [20, 21]. Natural Bond Orbital (NBO) analysis was then performed at the B3LYP/6-311+G** level by the NBO 3.1 program included in the GAUSSIAN 03 package of programs.

\section{Results and Discussion}

The results of DFT/B3LYP/6-311+G** contained in Table 3, 4 show that the highest activation energy calculated for substituted material is $25.12 \mathrm{kcal} / \mathrm{mol}$. According to the results in table 1,2 bond length C-S in the reaction of cis-2,5-three floro metyl Sulfolen (2), 2,5-threechlorometyl Sulfolen (3); 2,5three Bromo metyl Sulfolen (4) show that the shortest bond length and the strongest bond is C-S, which is supposed to break the more substituted 2,5 three Floro metyl. Highest Occupancy observed in C-S bond in the molecule 2 . This combination of factors makes 2,5-three Floro metyl Sulfolen (2) show a higher speed reaction because it has the lowest activation energy. This factor makes the compound cis-2,5-three Floro metyl Sulfolen (2) have high activation energy and lowest rate of reaction and the cis-2,5-three Bromo metyl Sulfolen [4] the lowest activation energy of cheletropic elimination reaction. Energy released in the reaction of cis-substituted will depend on the stability of reactant and instability of the product. High energy released in the cheletropic elimination reaction 2,5threechlorometyl Sulfolen (3).

As can be expected, the angle pressure and the large volume of substitution of 2,5-three Bromo metyl Sulfolen [4] are the main causes of molecules instability. But, if we calculate, the differential angular pressure response of the reactant and the product $d$ in the substitute $-2,5-$ three Floro metyl Sulfolen (2) and 2,5three Bromo metyl Sulfolen (4) we find that the difference in the combined 3 is 42 and in the combined 4 is 48 which increases the difference between the reactant and the product of 2,5threechlorometyl Sulfolen (3) reaction (Scheme 1). 


\begin{tabular}{|l|l|} 
Scheme 1. Angular strain in \\
the molecule,cis $2,5-$ \\
threechlorometyl Sulfolen(3) \\
product fraction
\end{tabular}

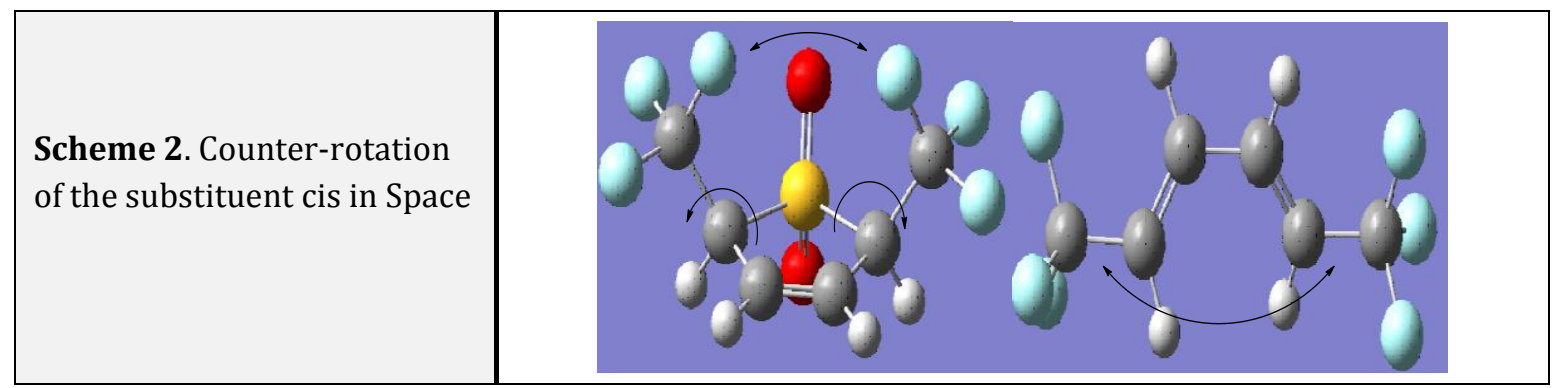

Table 1. DFT $/ 6-311+\mathrm{G}^{* *}$ calculated structural parameters of the ground states and transition stat of cis compounds

\begin{tabular}{|c|c|c|c|c|c|c|c|c|c|}
\hline Compounds & 2 & $\begin{array}{c}2 \rightarrow 2 / \\
X=C_{3}\end{array}$ & $2 /$ & 3 & $\begin{array}{l}3 \rightarrow 3 / \\
\mathrm{X}=\mathrm{Cl}_{3}\end{array}$ & $3 /$ & 4 & $\begin{array}{c}4 \rightarrow 4 / \\
\mathrm{X}=\mathrm{Br}_{3}\end{array}$ & $4 /$ \\
\hline \multicolumn{10}{|l|}{ Bond lengths } \\
\hline $\mathrm{C}_{1}-\mathrm{S}$ & 1.874 & 2.286 & ---- & 1.897 & 2.295 & --- & 1.906 & 2.289 & --- \\
\hline$C_{1-X}$ & 1.528 & 1.508 & 1.494 & 1.537 & 1.511 & 1.493 & 1.533 & 1.506 & 1.486 \\
\hline$C_{1}-C_{2}$ & 1.498 & 1.413 & 1.334 & 1.496 & 1.417 & 1.336 & 1.496 & 1.423 & 1.338 \\
\hline$C_{2}-C_{3}$ & 1.331 & 1.378 & 1.464 & 1.331 & 1.374 & 1.463 & 1.331 & 1.371 & 1.461 \\
\hline$C_{3}-C_{4}$ & 1.498 & 1.413 & 1.334 & 1.496 & 1.417 & 1.336 & 1.496 & 1.423 & 1.338 \\
\hline$C_{4-} S$ & 1.874 & 2.286 & ---- & 1.897 & 2.295 & --- & 1.906 & 2.289 & --- \\
\hline$C_{4}-\mathrm{x}$ & 1.52 & 1.508 & 1.494 & 1.537 & 1.511 & --- & 1.533 & 1.506 & 1.486 \\
\hline \multicolumn{10}{|l|}{ Bond angles $\left({ }^{\circ}\right)$} \\
\hline $\mathrm{S}-\mathrm{C}_{1}-\mathrm{C}_{2}$ & 114.06 & 97.28 & ---- & 103.595 & 96.515 & --- & 103.066 & 96.218 & --- \\
\hline $\mathrm{xC}-\mathrm{C}_{1}-\mathrm{C}_{2}$ & 118.37 & 119.57 & 123.24 & 115.672 & 120.766 & $\begin{array}{c}126.4 \\
95\end{array}$ & 116.043 & $\begin{array}{c}120.89 \\
6\end{array}$ & $\begin{array}{c}127.0 \\
56\end{array}$ \\
\hline $\mathrm{C}_{1}-\mathrm{C}_{2}-\mathrm{C}_{3}$ & 118.37 & 119.48 & 124.88 & 118.712 & 120.137 & $\begin{array}{c}124.5 \\
14\end{array}$ & 118.690 & $\begin{array}{c}120.36 \\
3\end{array}$ & $\begin{array}{c}124.8 \\
42\end{array}$ \\
\hline $\mathrm{C}_{2}-\mathrm{C}_{3}-\mathrm{C}_{4}$ & 104.07 & 119.48 & 124.88 & 118.712 & 120.137 & $\begin{array}{c}124.5 \\
14\end{array}$ & 118.690 & $\begin{array}{c}120.36 \\
3\end{array}$ & $\begin{array}{c}124.8 \\
42\end{array}$ \\
\hline $\mathrm{C}_{3}-\mathrm{C}_{4}-\mathrm{S}$ & 114.06 & 97.28 & ----- & 103.595 & 96.515 & --- & 103.66 & 96.218 & --- \\
\hline $\mathrm{C}_{3}-\mathrm{C}_{4}-\mathrm{Cx}$ & & 119.57 & 123.24 & 115.672 & 120.766 & $\begin{array}{c}126.4 \\
93\end{array}$ & 116.043 & $\begin{array}{c}120.89 \\
6\end{array}$ & $\begin{array}{c}127.5 \\
6\end{array}$ \\
\hline \multicolumn{10}{|l|}{$\begin{array}{c}\text { Torsio } \\
\text { angles }\left({ }^{\circ}\right)\end{array}$} \\
\hline $\mathrm{S}-\mathrm{C}_{1}-\mathrm{C}_{2}-\mathrm{C}_{3}$ & -4.56 & 39.30 & -- & -8.742 & 39.138 & --- & -12.375 & -38.807 & --- \\
\hline $\mathrm{F}_{3} \mathrm{C}-\mathrm{C}_{1}-\mathrm{C}_{2}-\mathrm{C}_{3}$ & 121.19 & 164.93 & -179.17 & 136.800 & 166.535 & $\begin{array}{c}- \\
179.0 \\
74\end{array}$ & -140.98 & $\begin{array}{c}- \\
1.66 .71 \\
4\end{array}$ & $\begin{array}{c}- \\
179.1 \\
16\end{array}$ \\
\hline $\mathrm{C}_{1}-\mathrm{C}_{2}-\mathrm{C}_{3}-\mathrm{C}_{4}$ & 0.000 & 0.000 & 34.41 & --- & 0 & $\begin{array}{c}33.73 \\
9\end{array}$ & 0 & 0 & $\begin{array}{c}30.66 \\
7\end{array}$ \\
\hline $\mathrm{C}_{2}-\mathrm{C}_{3}-\mathrm{C}_{4}-\mathrm{S}$ & 4.56 & -39.30 & --- & 8.742 & -39.138 & ---- & 12.375 & 38.807 & ---- \\
\hline $\mathrm{C}_{2}-\mathrm{C}_{3}-\mathrm{C}_{4}-\mathrm{Cx}$ & -121.19 & -164.93 & 179.17 & 136 & -136.53 & $\begin{array}{c}- \\
179.0 \\
81\end{array}$ & 140.98 & $\begin{array}{c}166.71 \\
4\end{array}$ & $\begin{array}{c}- \\
179.1 \\
16\end{array}$ \\
\hline
\end{tabular}


Table 2. DFT $/ 6-311+\mathrm{G}^{* *}$ calculated energies parameters of the ground states and transition stat of cis compounds

\begin{tabular}{|c|c|c|c|c|c|c|c|c|c|}
\hline Compounds & 2 & $\begin{array}{c}2 \rightarrow 2^{\prime} \\
\mathrm{X}=\mathrm{CF}_{3}\end{array}$ & $2^{\prime}$ & 3 & $\begin{array}{l}3 \rightarrow 3 / \\
\mathrm{X}=\mathrm{Cl}_{3}\end{array}$ & $3^{\prime}$ & 4 & $\begin{array}{c}4 \rightarrow 4^{\prime} \\
\mathrm{X}=\mathrm{Br}_{3}\end{array}$ & $4^{\prime}$ \\
\hline ZPE & 0.10731 & 0.10446 & $\begin{array}{c}0.1018 \\
0\end{array}$ & $\begin{array}{c}0.096 \\
61\end{array}$ & $\begin{array}{c}0.0939 \\
0\end{array}$ & $\begin{array}{c}0.0913 \\
9\end{array}$ & $\begin{array}{c}0.0923 \\
3\end{array}$ & $\begin{array}{c}0.0898 \\
2\end{array}$ & $\begin{array}{c}0.080 \\
70\end{array}$ \\
\hline $\mathrm{ZPE}^{\mathrm{c}}$ & 0.10097 & 0.09829 & $\begin{array}{c}0.0957 \\
88\end{array}$ & $\begin{array}{c}0.090 \\
906\end{array}$ & $\begin{array}{l}0.0883 \\
58\end{array}$ & $\begin{array}{c}0.0859 \\
95\end{array}$ & $\begin{array}{l}0.0868 \\
79\end{array}$ & $\begin{array}{c}0.0845 \\
15\end{array}$ & $\begin{array}{c}0.087 \\
410\end{array}$ \\
\hline Eel & $\begin{array}{c}- \\
1378.98 \\
3\end{array}$ & $\begin{array}{c}- \\
1378.96 \\
0\end{array}$ & $\begin{array}{c}- \\
1379.0 \\
02\end{array}$ & $\begin{array}{c}- \\
3541 .\end{array}$ & $\begin{array}{c}- \\
3541.0 \\
13\end{array}$ & $\begin{array}{c}- \\
3541.0 \\
54\end{array}$ & $\begin{array}{c}- \\
16224 . \\
546\end{array}$ & 16224. & $\begin{array}{c}- \\
1622 \\
4567\end{array}$ \\
\hline$E_{a}=E \mathrm{el}+Z P E^{c}$ & $\begin{array}{c}- \\
1378.88 \\
2\end{array}$ & $\begin{array}{c}- \\
1378.86 \\
2\end{array}$ & $\begin{array}{c}- \\
1378.9 \\
06\end{array}$ & $\begin{array}{c}- \\
3540 . \\
939\end{array}$ & $\begin{array}{c}- \\
3540.9 \\
24\end{array}$ & $\begin{array}{c}- \\
3540.9 \\
68\end{array}$ & $\begin{array}{c}- \\
16224 . \\
454\end{array}$ & $\begin{array}{c}- \\
16224 . \\
446\end{array}$ & $\begin{array}{c}- \\
1622 \\
4.479\end{array}$ \\
\hline$\Delta E^{\mathrm{b}} \mathrm{a}($ Hartree $)$ & $\begin{array}{c}0.02469 \\
9\end{array}$ & $\begin{array}{c}0.04422 \\
7\end{array}$ & 0.00 & $\begin{array}{c}0.028 \\
204\end{array}$ & $\begin{array}{c}0.0432 \\
87\end{array}$ & 0.00 & 0.00 & $\begin{array}{c}0.0331 \\
88\end{array}$ & 0.00 \\
\hline$\Delta E_{a}\left(\mathrm{kcal} \mathrm{mol}^{-1}\right)$ & $\begin{array}{c}15.4990 \\
6\end{array}$ & $\begin{array}{c}27.7528 \\
8\end{array}$ & 0.00 & $\begin{array}{c}17.69 \\
836\end{array}$ & 27.162 & 0.00 & 0.00 & 20.825 & 0.00 \\
\hline 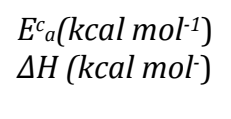 & & $\begin{array}{r}12.253 \\
-15.499\end{array}$ & & & $\begin{array}{c}9.464 \\
- \\
17.698\end{array}$ & & & $\begin{array}{c}8.169 \\
- \\
12.656\end{array}$ & \\
\hline
\end{tabular}

The resultant instability of the bond length and large size $\mathrm{C}-\mathrm{CBr}_{3}$ and electronegative substituent is less than the shorter bond length and small volume $\mathrm{C}-\mathrm{CCl}_{3}$, so that reactant is expected to be more stable than the substituted $\mathrm{CCl}_{3}$ 2,5-three Bromo metyl Sulfolen (4). Reaction pathways are shown in Figures 2. The B3LYP/6- 311+G** calculated energies and calculated structural parameters are given in Tables 1 , 2. scheme 2 Counter-rotation of the substituent cis in Space.

Activation energy changes calculated by DFT/B3LYP/6-311+G** for trans compounds show that Trans-2,5- three chloro metyl Sulfolen (7) has the highest activation energy and the lowest rate of reaction.

Muliken changing both substituted indicating trans-form stericl effects causes amounts of substituted charge to be different. These steric and electronic effects on the reaction process revealed that free energy change for the three transsubstituted compounds indicate that the largest trans-substituted compounds. This may be due to the large volume of brom in 2,5- three Bromo metyl Sulfolen (7) which can lead to instability of reactant and products. After elimination of $\mathrm{SO}_{2}$ in response to the energy difference between the products, the material reaches the maximum value of stability. This results in greater spatial effects and can be seen in the 
electron impact and shows that the trans2,5- three Bromo metyl Sulfolen (7) is released due to the great instability and higher activition energy. Reaction pathways are shown in Figures 3. The B3LYP/6$311+\mathrm{G}^{* *}$ calculated energies and calculated structural parameters are given in Tables 3, 4.

Table3. DFT/6-311+G** calculated structural parameters of the ground states and transition stat of trans compounds

\begin{tabular}{|c|c|c|c|c|c|c|c|c|c|}
\hline Compounds & 5 & $\begin{array}{c}5 \rightarrow 5 / \\
\mathrm{X}=\mathrm{CF}_{3}\end{array}$ & $5^{\prime}$ & 6 & $\begin{array}{l}3 \rightarrow 3 / \\
\mathrm{X}=\mathrm{Cl}_{3}\end{array}$ & $6 /$ & 7 & $\begin{array}{c}7 \rightarrow 7 / \\
\mathrm{X}=\mathrm{Br}_{3}\end{array}$ & $7 /$ \\
\hline \multicolumn{10}{|l|}{$\begin{array}{l}\text { Bond } \\
\text { lengths }\end{array}$} \\
\hline $\mathrm{C}_{1}-\mathrm{S}$ & 1.877 & 2.377 & ---- & 1.894 & 2.376 & --- & 1.8964 & 2.376 & --- \\
\hline$C_{1}-C X_{3}$ & 1.528 & 1.511 & 1.500 & 1.539 & 1.515 & 1.495 & 1.5337 & 1.5075 & 1.4955 \\
\hline$C_{1}-C_{2}$ & 1.498 & 1.414 & 1.337 & 1.499 & 1.418 & 1.335 & 1.498 & 1.4238 & 1.344 \\
\hline$C_{2}-C_{3}$ & 1.330 & 1.405 & 1.465 & 1.330 & 1.382 & 1.462 & 1.330 & 1.380 & 1.459 \\
\hline$C_{3}-C_{4}$ & 1.498 & 1.413 & 1.333 & 1.498 & 1.403 & 1.341 & 1.499 & 1.4069 & 1.338 \\
\hline$C_{4-} S$ & 1.877 & 3.029 & --- & 1.890 & 2.433 & ---- & 1.900 & 2.447 & --- \\
\hline$C_{4-} \mathrm{CX}_{3}$ & 1.528 & 1.507 & 1.495 & 1.536 & 1.507 & 1.502 & 1.536 & 1.500 & 1.4882 \\
\hline \multicolumn{10}{|l|}{$\begin{array}{c}\text { Bond } \\
\text { angles }\left({ }^{\circ}\right)\end{array}$} \\
\hline $\mathrm{S}-\mathrm{C}_{1}-\mathrm{C}_{2}$ & $\begin{array}{c}104.4 \\
8\end{array}$ & 97.28 & --- & $\begin{array}{c}102.77 \\
1\end{array}$ & 93.281 & ---- & 102.809 & $\begin{array}{c}92.969 \\
6\end{array}$ & ---- \\
\hline $\mathrm{X}_{3} \mathrm{C}-\mathrm{C}_{1}-\mathrm{C}_{2}$ & $\begin{array}{c}113.7 \\
4\end{array}$ & 119.57 & 127.14 & $\begin{array}{c}115.41 \\
2\end{array}$ & $\begin{array}{c}128.35 \\
4\end{array}$ & $\begin{array}{c}125.93 \\
6\end{array}$ & 115.720 & $\begin{array}{c}128.75 \\
4\end{array}$ & $\begin{array}{c}131 . \\
7928\end{array}$ \\
\hline $\mathrm{C}_{1}-\mathrm{C}_{2}-\mathrm{C}_{3}$ & $\begin{array}{c}118.3 \\
3\end{array}$ & 119.48 & 130.02 & $\begin{array}{c}118.70 \\
7\end{array}$ & $\begin{array}{c}126.95 \\
8\end{array}$ & $\begin{array}{c}126.45 \\
8\end{array}$ & $\begin{array}{c}118.656 \\
8\end{array}$ & $\begin{array}{c}127.49 \\
9\end{array}$ & 134.291 \\
\hline $\mathrm{C}_{2}-\mathrm{C}_{3}-\mathrm{C}_{4}$ & $\begin{array}{c}118.3 \\
3\end{array}$ & 119.48 & 126.22 & $\begin{array}{c}118.26 \\
6\end{array}$ & $\begin{array}{c}124.07 \\
4\end{array}$ & $\begin{array}{c}133,49 \\
9\end{array}$ & 119.11 & $\begin{array}{c}124.48 \\
7\end{array}$ & 126.726 \\
\hline $\mathrm{C}_{3}-\mathrm{C}_{4}-\mathrm{S}$ & $\begin{array}{c}104.4 \\
8\end{array}$ & 97.28 & ----- & $\begin{array}{c}103.04 \\
7\end{array}$ & 93.443 & ----- & 102.575 & 92.801 & ---- \\
\hline $\mathrm{C}_{3}-\mathrm{C}_{4}-\mathrm{CX}_{3}$ & $\begin{array}{c}114.7 \\
3\end{array}$ & 119.57 & 122.73 & $\begin{array}{c}114.45 \\
1 \\
\end{array}$ & $\begin{array}{c}121.16 \\
6\end{array}$ & $\begin{array}{c}130.92 \\
3 \\
\end{array}$ & 115.944 & $\begin{array}{c}121.50 \\
0\end{array}$ & 126.414 \\
\hline \multicolumn{10}{|l|}{$\begin{array}{c}\text { Torsio } \\
\text { angles }\left({ }^{\circ}\right)\end{array}$} \\
\hline $\mathrm{S}-\mathrm{C}_{1}-\mathrm{C}_{2}-\mathrm{C}_{3}$ & $\begin{array}{c}- \\
4.567\end{array}$ & 37.156 & --- & $\begin{array}{c}- \\
11.955\end{array}$ & 35.975 & --- & 12.264 & 35.667 & ---- \\
\hline $\mathrm{X}_{3} \mathrm{C}-\mathrm{C}_{1}-\mathrm{C}_{2}-\mathrm{C}_{3}$ & $\begin{array}{c}124.1 \\
65\end{array}$ & $\begin{array}{c}- \\
69.996\end{array}$ & 2.097 & $\begin{array}{c}115.01 \\
2\end{array}$ & $\begin{array}{c}- \\
77.357\end{array}$ & $\begin{array}{c}176.81 \\
0\end{array}$ & 139.956 & $\begin{array}{c}- \\
79.406\end{array}$ & 3.0024 \\
\hline $\mathrm{C}_{1}-\mathrm{C}_{2}-\mathrm{C}_{3}-\mathrm{C}_{4}$ & $\begin{array}{c}- \\
0.080\end{array}$ & -0.384 & 41.007 & $\begin{array}{c}- \\
0.9016\end{array}$ & $\begin{array}{c}- \\
0.1758\end{array}$ & 39.731 & -1.601 & 0.1954 & 39.355 \\
\hline $\mathrm{C}_{2}-\mathrm{C}_{3}-\mathrm{C}_{4}-\mathrm{S}$ & 0.572 & $\begin{array}{c}- \\
26.170\end{array}$ & ---- & 0.572 & $\begin{array}{c}- \\
34.805\end{array}$ & --- & -9.975 & $\begin{array}{c}- \\
34.706 \\
6\end{array}$ & ---- \\
\hline $\mathrm{C}_{2}-\mathrm{C}_{3}-\mathrm{C}_{4}-\mathrm{CX}_{3}$ & $\begin{array}{c}125.1 \\
84\end{array}$ & $\begin{array}{c}- \\
159.89 \\
4\end{array}$ & $\begin{array}{c}177.90 \\
1\end{array}$ & $\begin{array}{c}140.31 \\
9\end{array}$ & $\begin{array}{c}- \\
160.59 \\
2\end{array}$ & 2.252 & 117.854 & $\begin{array}{c}- \\
160.64\end{array}$ & $\begin{array}{c}175.013 \\
8\end{array}$ \\
\hline
\end{tabular}


Table 4. DFT $/ 6-311+G^{* *}$ calculated energies parameters of the ground states and transition stat of trans of compounds

\begin{tabular}{|c|c|c|c|c|c|c|c|c|c|}
\hline Compounds & 5 & $5 \rightarrow 5 /$ & $5 /$ & 6 & $6 \rightarrow 6 /$ & $6 /$ & 7 & $7 \rightarrow 7 /$ & $7 /$ \\
\hline ZPE & $\begin{array}{c}0.1072 \\
5\end{array}$ & $\begin{array}{c}0.1042 \\
5\end{array}$ & $\begin{array}{c}0.1019 \\
9\end{array}$ & $\begin{array}{c}0.0966 \\
8\end{array}$ & $\begin{array}{c}0.0934 \\
2\end{array}$ & $\begin{array}{c}0.0915 \\
6\end{array}$ & $\begin{array}{c}0.0923 \\
9\end{array}$ & $\begin{array}{c}0.0890 \\
9\end{array}$ & 0.08745 \\
\hline $\mathrm{ZPE}^{\mathrm{c}}$ & $\begin{array}{c}0.1009 \\
1\end{array}$ & $\begin{array}{c}0.0980 \\
9\end{array}$ & $\begin{array}{c}0.0959 \\
6\end{array}$ & $\begin{array}{c}0.0909 \\
6\end{array}$ & $\begin{array}{c}0.0878 \\
9\end{array}$ & $\begin{array}{c}0.0861 \\
5\end{array}$ & $\begin{array}{c}0.0869 \\
3\end{array}$ & $\begin{array}{c}0.0838 \\
3\end{array}$ & 0.08228 \\
\hline Eel & $\begin{array}{c}- \\
1378.9 \\
82\end{array}$ & $\begin{array}{c}- \\
1378.9 \\
51\end{array}$ & $\begin{array}{c}- \\
1378.9 \\
96\end{array}$ & $\begin{array}{c}- \\
3541.0 \\
30\end{array}$ & $\begin{array}{c}- \\
3540.9 \\
96\end{array}$ & $\begin{array}{c}- \\
3541.0 \\
44\end{array}$ & $\begin{array}{c}- \\
16224 . \\
546\end{array}$ & $\begin{array}{c}- \\
16224 . \\
513\end{array}$ & $\begin{array}{c}- \\
16224.5 \\
62\end{array}$ \\
\hline$E_{a}=E \mathrm{el}+Z P E^{c}$ & $\begin{array}{c}- \\
1378.8 \\
81\end{array}$ & $\begin{array}{c}- \\
1378.8 \\
53\end{array}$ & $\begin{array}{c}- \\
1378.9 \\
00\end{array}$ & $\begin{array}{c}- \\
3540.9 \\
39\end{array}$ & $\begin{array}{c}- \\
3540.9 \\
09\end{array}$ & $\begin{array}{c}- \\
3540.9 \\
58\end{array}$ & $\begin{array}{c}- \\
16224 . \\
459\end{array}$ & $\begin{array}{c}- \\
16224 . \\
429\end{array}$ & $\begin{array}{c}- \\
16224.4 \\
80\end{array}$ \\
\hline$\Delta E^{\mathrm{b}}{ }_{\mathrm{a}}($ Hartree $)$ & $\begin{array}{c}0.0187 \\
84\end{array}$ & $\begin{array}{c}0.0470 \\
23\end{array}$ & 0.00 & $\begin{array}{c}0.0185 \\
06\end{array}$ & $\begin{array}{c}0.0494 \\
14\end{array}$ & 0.00 & $\begin{array}{c}0.0207 \\
11\end{array}$ & $\begin{array}{c}0.0503 \\
92\end{array}$ & 0.00 \\
\hline $\begin{array}{l}\Delta E_{a}\left(\mathrm{kcal} \mathrm{mol}^{-1}\right) \\
E_{a}^{c}\left(\mathrm{kcal} \mathrm{mol}^{-1}\right) \\
\Delta H\left(\mathrm{kcal} \mathrm{mol}^{-}\right)\end{array}$ & 11.786 & $\begin{array}{c}29.506 \\
17.719 \\
- \\
11.786\end{array}$ & 0.00 & 11.612 & $\begin{array}{c}31.007 \\
19.394 \\
- \\
11.612 \\
8\end{array}$ & 0.00 & 12.995 & $\begin{array}{c}31.621 \\
18.625 \\
- \\
12.995\end{array}$ & 0.00 \\
\hline
\end{tabular}

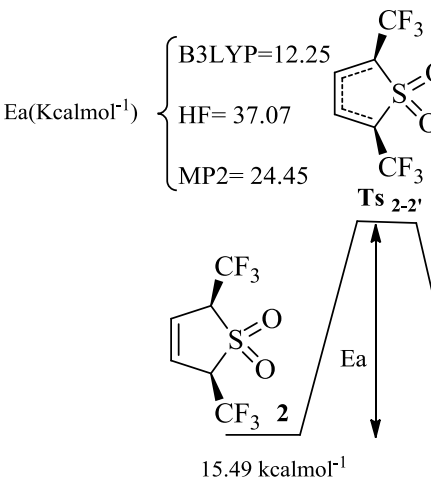

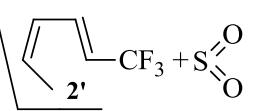

$0.00 \mathrm{kcalmol}^{-1}$
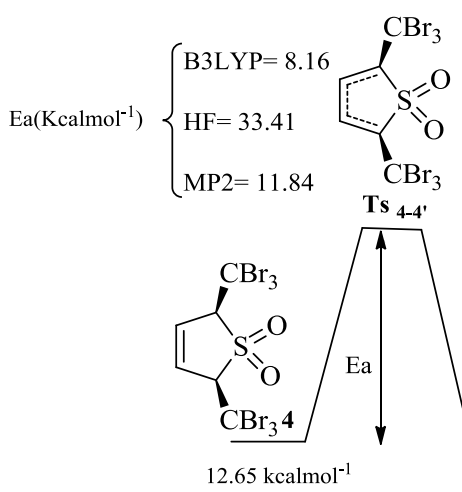

Fig 2. Energies diagram for compounds 2,4 and 5 


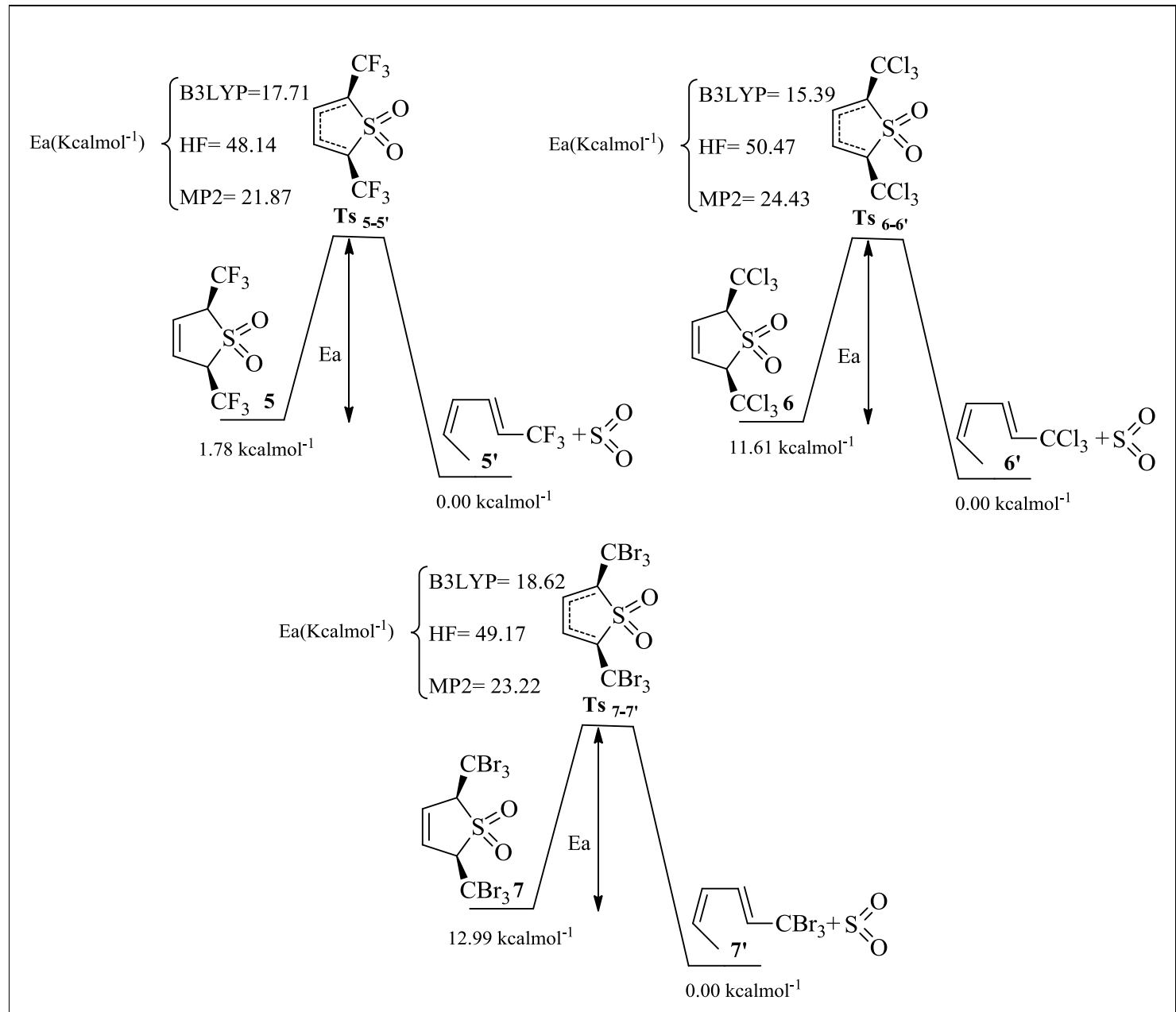

Figure 3. Energies diagram for compounds 5,6 and 7

\section{Conclusions}

The results of quantum mechanical and theoretical calculations early in the DFT/B3LYP/6-311+G** and MP2/6$311+\mathrm{G}^{* *}$ and electronic effects on the reaction rate on cheletropic reaction $\mathrm{HF} / 6$ $311+G^{* *} 2-5$ dihydro thiophene sulfone showed that the endothermic reaction energy level of the products is higher than the reactant. Activation energy of 20.18 $\mathrm{kcal} / \mathrm{mol}$ is lower than the estimated value so the elimination speed is minimal. Levels of theory calculations on three levels with a combination of spatial substituted cis showed that the lowest rate of reaction 2,5three Floro metyl Sulfolen (2) with the substituent is electronegative. Theoretical calculations on three combinations of substituted trans showed that the lowest rate of reaction is related to trans- 2,5- three Bromo metyl Sulfolen 
(7). In elimination cheletropic reaction with substituted trans and counter rotation electron effects and steric effects of product have direct effects on the reaction rate. Free energy of reaction for the synthesis of trans- 2,5- three Bromo

\section{References}

[1]. Ullmann's Encyclopedia of Industrial Chemistry, Wiley, 2011.

[2]. E.V. Anslyn, D.A. Dougherty, Modern Phys. Org. Chem., University Science Books, 2006.

[3]. I. Fleming, Frontier Orbitals and Organic Chemistry Reactions. Wiley, 1976.

[4]. V. Polo, J. Andre's, S. Berski, L.R. Domingo, B. Silvi, J. Phys. Chem. A., 2008, 112, 7128-7136.

[5]. S. Berski, J. Andre's, B. Silvi, L.R. Domingo, J. Phys. Chem. A., 2006, 110, 13939-13947.

[6]. M. Ríos-Gutie'rrez, L.R. Domingo, P. Pe'rez, RSC Adv., 2015, 84797-84809.

[7]. L.R. Domingo, P. Pe'rez, J.A. Sa'ez, Tetrahedron, 2013, 69, 107-114.

[8]. S. Jameh-Bozorghi, M. Darvishpour, S. Mostghiman, Z. Javanshir, Int. J. Electrochem. Sci., 2011, 6, 4891-4899.

[9]. S. Jameh-Bozorghi, M. Darvishpour, S. Mohammadi, Z. Javanshir, Int. J. Electrochem. Sci., 2011, 6, 5031-5037.

[10]. Z. Javanshir, S. Jameh-Bozorghi, A.R Namdari, Cur Sci., 2017, 112, 743-749. metyl Sulfolen (7) is $99.12 \mathrm{kcal} / \mathrm{mol}$. The largest volume brominated high pressure angle causes a high energy difference between the material and the product.

[11]. S. Jameh-Bozorghi, Z. Javanshir, D. Nori Shargh, J. Adv. Chem.., 2013, 5, 614-625.

[12]. A. Ghaempanah, S. Jameh-Bozorghi, M. Darvishpour, M.H. Fekri, Int. J. Electrochem. Sci., 2012, 7, 6127-6133.

[13]. L.R. Domingo, M.J. Aurella, P. Pe'rez, Org. Biomol. Chem., 2014, 12, 7581-7590.

[14]. L.R. Domingo, M. Ríos-Gutie'rrez, P. Pe'rez, Tetrahedron, 2015, 71, 2421-2427.

[15]. P. Pe'rez, L.R. Domingo, Eur. J. Org. Chem., 2015, 2826-2834.

[16]. A.K. Nacereddine, C. Sobhi, A. Djerourou, M. Rı'os-Gutie'rrez, L.R. Domingoc, RSC Adv., 2015, 5, 99299-99311. [17]. W. Koch, M.C. Holthausen, A Chemist's Guide to Density Functional Theory; WileyVCH, 2001.

[18]. P. Jaque, J.V. Correa, F.D. Proft, A. ToroLabbe', P. Geerlings, Can. J. Chem., 2010, 88, 858-865.

[19]. M.J. Frisch, G.W. Trucks, H.B. Schlegel, G.E. Scuseria, M.A. Robb, J.R. Cheeseman, J. A. Montgomery, T. Vreven, K.N. Kudin, J.C. Burant, J.M. Millam, S.S. Iyengar, J. Tomasi, V. Barone, B. Mennucci, M. Cossi, G. Scalmani, 
N. Rega, G.A. Petersson, H. Nakatsuji, M. Cui, A.G. Baboul, S. Clifford, J. Cioslowski, B.B. Hada, M. Ehara, K. Toyota, R. Fukuda, J. Stefanov, G. Liu, A. Liashenko, P. Piskorz, I. Hasegawa, M. Ishida, T. Nakajima, Y. Honda, Komaromi, R.L. Martin, D.J. Fox, T. Keith, O. Kitao, H. Nakai, M. Klene, X. Li, J.E. Knox, H. $\quad$ M.A. Al-Laham, C.Y. Peng, A. Nanayakkara, M. P. Hratchian, J.B. Cross, V. Bakken, C. Adamo, Challacombe, P.M.W. Gill, B. Johnson, W. J. Jaramillo, R. Gomperts, R.E. Stratmann, O. Chen, M.W. Wong, C. Gonzalez, J.A. Pople, Yazyev, A.J. Austin, R. Cammi, C. Pomelli, J.W. $\quad$ Gaussian, Inc., Wallingford CT, 2004.

Ochterski, P.Y. Ayala, K. Morokuma, G.A. [20]. C. Peng, H.B. Schlegel, Israel J. Chem., Voth, P. Salvador, J.J. Dannenberg, V.G. 1993, 33, 449-454.

Zakrzewski, S. Dapprich, A.D. Daniels, M.C. [21]. C. Peng, P.Y. Ayala, H.B. Schlegel, M.J. Strain, O. Farkas, D.K. Malick, A.D. Rabuck, K. $\quad$ Frisch, J. Comp. Chem., 1996, 17, 49-56.

Raghavachari, J.B. Foresman, J.V. Ortiz, Q.

How to cite this manuscript: Zahra Javanshir*, Saeed Jameh-Bozorghi, Parasto Peyki, DFT Calculations of the Neighboring Groups Effects on Cheletropic Reaction of 2,5Dihydrothiophene Sulfone, Adv. J. Chem. A, 2018, 1(2), 117-126. 